\title{
Automated universal chip platform for fluorescence based cellular assays
}

\author{
F. Schmieder ${ }^{1}$, S. Schmieder ${ }^{1}$, R. Eger ${ }^{1}$, S. Friedrich ${ }^{2}$, A. Werner ${ }^{2}$, N. Danz ${ }^{3}$, U. Marx ${ }^{4}$, F. Sonntag ${ }^{1}$ \\ ${ }^{1}$ Fraunhofer Institute for Material and Beam Technology IWS, Dresden, Germany, frank.sonntag@iws.fraunhofer.de \\ ${ }^{2}$ TU Dresden, Institute of Food Technology and Bioprocess Engineering, Dresden, Germany \\ ${ }^{3}$ Fraunhofer Institute for Applied Optics and Precision Engineering IOF, Jena, Germany \\ ${ }^{4}$ TU Berlin, Institute for Medical Biotechnology, Berlin, Germany
}

\begin{abstract}
The advantage of cell based assays used as biosensors is the direct access to hardly obtainable parameters like toxicity, mutagenicity and pharmacological effectiveness. Within the last few years we established a micro fluidic platform including a peristaltic micro pump as well as several valves, manifolds and micro channels [1]. For optical online monitoring the micro fluidic system is bonded to a glass slide. Furthermore the biochip is fixed on an electrically heated support. The pneumatically actuated peristaltic pump as well as the temperature control is performed by a control device. For the fluorescence based online monitoring a robotic guided fluorescence measurement module was developed, which supports the detection of fluorescence in microtiter plates and microfluidic systems. This measurement module allows the fluorescence detection of two different excitation / detection wavelengths (480/530 nm and $570 / 620 \mathrm{~nm})$ and was successfully characterised using EGFP and Rhodamine 6G. Additionally three cell based assays with bacterial, yeast and human cells were characterized.
\end{abstract}

\section{Introduction}

Cell based assays are a major area in biosensor research. The significant advantage of whole cell assays in comparison to biomolecules results from the direct access to hardly obtainable parameters such as toxicity, mutagenicity and pharmacological effectiveness. The main fields of application of cell based assays can be found in pharmaco- and toxicokinetics [2], biomedicine [3], environmental monitoring [4] as well as in basic research such as cell physiology and stem cell sciences. Among cell based assays, reporter gene assays became more and more important, because they are very flexible in varying the properties of the biological component by genetic modification. Caused by this big variety of applications a universal system is needed to adapt all these different assays to one platform. Basically all cell based biosensors consist of an immobilised biological component at the edge of a transducing device like an electrode or an optical component like a transparent surface and a photodetector. The biggest advantage of optical measurements is the ability to detect low quantities of target molecules without direct contact to the probe. Among those photometric detection approaches fluorescence measurement is the most common method. One reason for that is the wide range of fluorescent dyes that allows the detection of target molecules at almost any wavelength from UV up to near Infrared. Moreover only small sample volumes are needed. Nevertheless in most cases fluorescence dyes are designed for special excitation wavelengths, which are available in almost any fluorescence microscope or flow cytometer. Using these benefits of fluorescence biosensors many applications for cell based assays were built up in the past. Most of them are specified for single tasks like laboratory measurements in cuvettes or the measurement of fluorescence in microtiter plates. In this paper we describe a fluorescence measurement system that is build for the automated measurement in microtiter plates or microreactors and that is tested with different applications in environmental monitoring, pharmacological effectiveness and biomedicine. Therefore an excitation system with two common excitation wavelengths is characterised and used. The focus of the subsequent applications lies on cell based assays, especially suitable reporter gen assays using EGFP.

\section{Methods}

\subsection{Chip system}

The flow cell contains the microfluidic system, which consists of channels, micro pumps, valves and a reaction chamber for the immobilised cells. Moreover a metal connector plate provides efficient thermal transport into the micro reactor. The chip system is built out of several layers and was formed by moulding a polydimethylsiloxane (PDMS) cell, which is casted from a master die, onto a polymeric cover plate. Afterwards a glass slide was bonded to the PDMS layer in order to cover the upper side of the chip. Image 1 shows the structure of the microfluidic system. The input (1) and the first membrane pump for the distribution of the medium and other fluids (2) are located at the right hand side. The fluid contained in the measuring chamber (6) at the left side is circulated by the internal micro pump (5). For rinsing the first membrane pump and the input the side port (3) can be opened by a further valve (4). 


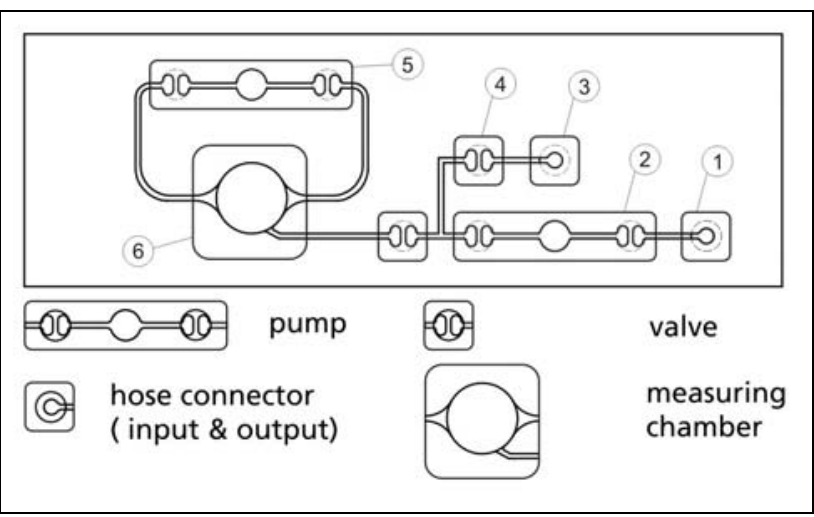

Image 1 General structure of the microfluidic system.

\subsection{Fluorescence measurement}

The fluorescence measurement was performed by a system containing several modules. For the excitation two LEDs with peak wavelength at 467 and $590 \mathrm{~nm}$ where integrated into a lens system and used as the light source. The excitation wavelength is separated from the fluorescence signal by a multibandpass filter set (530/25 and 635/25). Fluorescence is coupled into a fibre output for external spectral analysis. Image 2 shows a sketch of the setup.

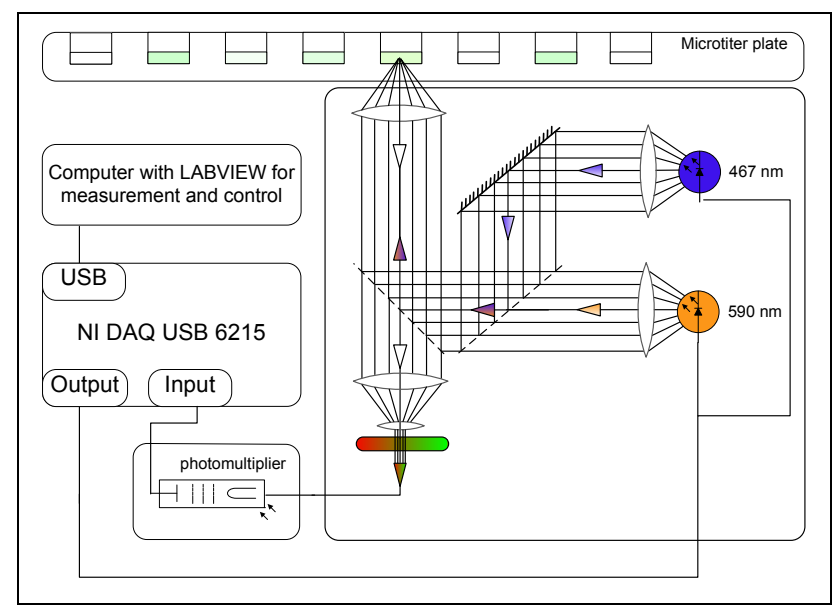

Image 2 Schematic setup of the fluorescence measurement system.

For the detection of the fluorescence signal a photomultiplier (Hamamatsu H3165-10) with adjustable amplification voltage of up to $1000 \mathrm{~V}$ was used. As indicated in image 2, the data acquisition module NI DAQ USB 6215 was used to control the LEDs for fluorescence excitation and to convert the analog input signal of the photomultiplier. A LABVIEW application was generated to set the digital output signals and to record the incoming fluorescence signal and write it into an Excel file.

\subsection{Robotic platform}

The robotic platform for the automated handling of liquid samples and media was a Nanoplotter 2 by GeSiM. It was used to fill the wells with medium and cell suspension and to dose the antibiotic solutions and the methanol into the assays.

\section{$2.4 \quad$ Cellular assays}

\subsubsection{Antibiotic resistance assay based on EGFP producing $\boldsymbol{P}$. fluorescens cells}

The Pseudomonas fluorescens (SBW25 GFP-ASV) strain was obtained from the School of Contemporary Sciences, University of Albertay Dundee and maintain on NA-agar plates $(8 \mathrm{~g} / \mathrm{L})$. For fluorescence measurements in microtiter plates or in the chip system the stock culture was inoculated overnight in $20 \mathrm{~mL} \mathrm{NB}$-medium containing gentamycinsulfate $(50 \mu \mathrm{g} / \mathrm{mL})$ at $30^{\circ} \mathrm{C}$ and $260 \mathrm{rpm}$. For fluorescence measurements the $P$. fluorescens cells were spun down at 10000 ref for 90 seconds and resuspended in pseudomonas minimal medium (pseudomonas saccarophilia medium [5]) containing per liter: $4.8 \mathrm{~g} \mathrm{Na}_{2} \mathrm{HPO}_{4}$; $4.4 \mathrm{~g} \mathrm{KH}_{2} \mathrm{PO}_{4} ; 1 \mathrm{~g} \mathrm{NH}_{4} \mathrm{Cl} ; 0.5 \mathrm{~g} \mathrm{MgSO}_{4} * 7 \mathrm{H}_{2} \mathrm{O} ; 0.05 \mathrm{~g}$ Ammonium ferric citrate; $1 \mathrm{~g}$ glucose. Furthermore the medium contained $50 \mu \mathrm{g} / \mathrm{mL}$ gentamycinsulfate. Afterwards the OD600 of the suspension was adjusted and the cells where distributed to a microtiter plate. During the measurement time the pseudomonas culture was incubated at $30^{\circ} \mathrm{C}$ and $200 \mathrm{rpm}$. To evaluate the antibiotic resistance of the strain ampicillin $(10 \mu \mathrm{g} / \mathrm{mL})$, chloramphenicol $(25 \mu \mathrm{g} / \mathrm{mL})$, kanamycin $(50 \mu \mathrm{g} / \mathrm{mL})$ und tetracyclin $(15 \mu \mathrm{g} / \mathrm{mL})$ where added to different wells at the beginning of the tests. As a control for antibiotic resistance an antibiogram was performed with all 4 antibiotics as well as gentamycin.

\subsubsection{Yeast cell based EGFP-reporter gen assay}

Pichia pastoris (GS115:AOX1-EGFP) was obtained from Institute of genetics, Technische Universität Dresden and maintained on YPD-agar plates $(10 \mathrm{~g} / \mathrm{L}$ yeast extract, $20 \mathrm{~g} / \mathrm{L}$ peptone, $20 \mathrm{~g} / \mathrm{L}$ dextrose). For fluorescence measurements in microtiter plates or in the chipsystem the stock culture was inoculated overnight in $20 \mathrm{~mL}$ YPD-medium $(1 \mathrm{~g} / \mathrm{L}$ yeast extract, $2 \mathrm{~g} / \mathrm{L}$ peptone, $2 \mathrm{~g} / \mathrm{L}$ dextrose) at $30{ }^{\circ} \mathrm{C}$ and $260 \mathrm{rpm}$. For fluorescence measurements the $P$. pastoris cells were spun down at 10000 rcf for 60 seconds, resuspended in yeast minimal medium ( containing per liter: Yeast Nitrogen Base $6.7 \mathrm{~g}$, glucose $1 \mathrm{~g}$ ), the OD600 of the suspension was adjusted and the suspension was distributed to the microtiter plate. During the measurement time the P. pastoris culture was incubated at $30^{\circ} \mathrm{C}$ and $200 \mathrm{rpm}$. At the beginning of the measurement different amounts of methanol $(100 \%)$ were added to the solution, so the final concentrations of methanol in the wells were $0.5 \%, 0.1 \%, 0.05 \%, 0.01 \%$ and $0.005 \%(\mathrm{v} / \mathrm{v})$.

\subsubsection{Human HepG2 cell line stained with Cell Tracker Red and Green}

Cells where purchased from Institut für angewandte Zellkultur, Dr. Toni Lindl GmbH and cultivated in DMEM containing $10 \%(\mathrm{v} / \mathrm{v}) \mathrm{FCS}$ and $1 \%(\mathrm{v} / \mathrm{v}) \mathrm{P} / \mathrm{S}$ at $37^{\circ} \mathrm{C}$ and $5 \% \mathrm{CO}_{2}$. Afterwards they were stained with either Celltracker Green CMFDA or Celltracker Red CMTPX and fixated on a BD Falcon ${ }^{\mathrm{TM}}$ 4-well culture slide and stored 
in ClearMount ${ }^{\mathrm{TM}}$ mounting solution at $4{ }^{\circ} \mathrm{C}$. For the detection of the thickness of the cell layer, the fixated cell layer was observed under the light microscope. Afterwards fluorescence was measured at the same position.

\section{$3 \quad$ Results}

\subsection{Evaluation of the fluorescence measure- ment}

The dyes EGFP (excitation wavelength $489 \mathrm{~nm} /$ emission wavelength $509 \mathrm{~nm}$ ) and Rhodamine 6G (excitation wavelength $489 \mathrm{~nm} /$ emission wavelength $563 \mathrm{~nm}$ ) where used to evaluate the system. To prove these spectral characteristics of the fluorescent dyes within solutions, a 3D-scan was performed in a photospectrometer (Hitachi F4500). The amplification of the photomultiplier was adjusted by measuring different concentrations of the fluorescent dyes and comparing their relative signals. Image 3 shows the fluorescence signal as a function of the EGFP-concentration at different amplifications. As one can see the signal increases with higher amplifications but becomes nonlinear at very high amplifications. That appears in case of saturating the amplification, when an increasing fluorescence signal will not yield higher output. Similar results were observed by using Rhodamine 6G.

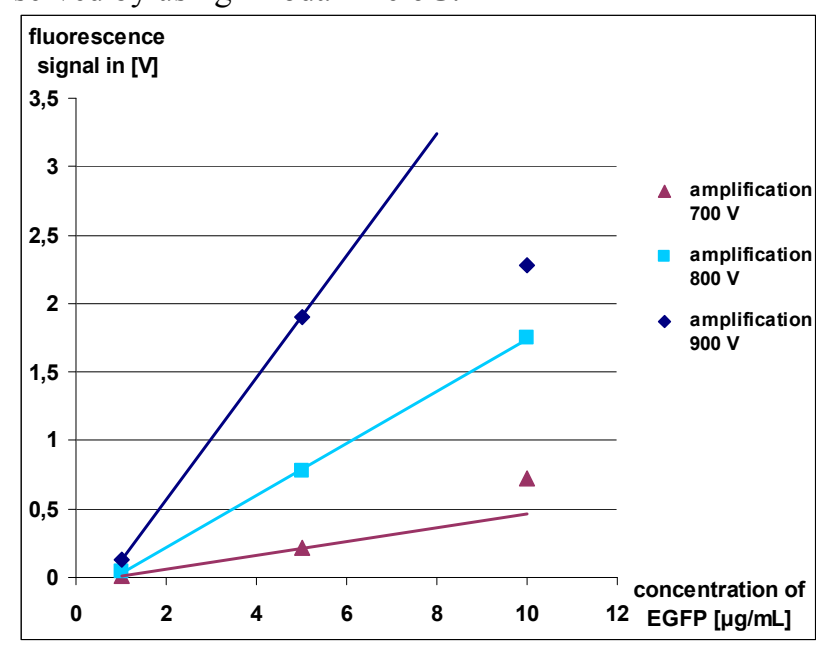

Image 3 Fluorescence signal as a function of concentration.

In result the general amplification for further measurements was set to $800 \mathrm{~V}$. Nevertheless other amplifications can be used if required by the fluorescent assay. Referring to that amplification the inherent fluorescence of the microtiter plates, the growth media and the nonfluorescent cells was measured and compared to the results of the measurements with fluorescent cells. The range of the output voltage was detected by measurements with water for the lower limit $(1.3 \mathrm{mV})$ and daylight $(3.5 \mathrm{~V})$ for the upper limit.

\subsection{Application of the cellular assays}

\subsubsection{Antibiotic resistance assay using $P$. fluo- rescens}

The measurement of the antibiotic resistance showed almost similar results as the antibiogram performed before those tests. Image 4 shows these results. The antibiogram as well as the fluorescent assay showed antibiotic resistance against ampicillin.

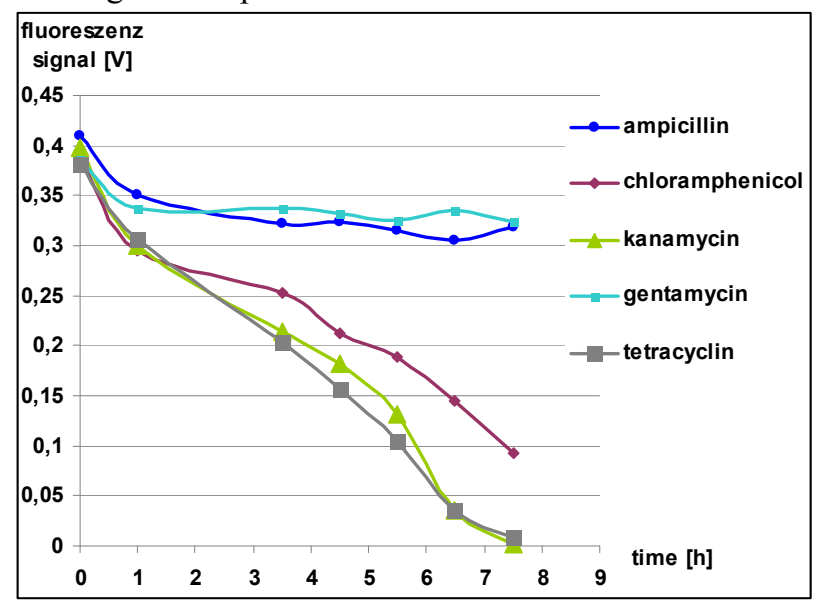

Image 4 Fluorescence signal of $P$. fluorescens inhibited by several antibiotics.

During the antibiotic inhibited cultivation the fluorescence signal was dropping with kanamycin, tetracyclin and chloramphenicol. Thereby the fastest results were observed with kanamycin and tetracyclin. Chloramphenicol showed also adorable results. The use of the pseudomonas minimal medium showed the excepted effect of degrading the autofluorescence of the cellular assay. A main problem in cultivating the pseudomonas cells within the microtiter plate was the oxygen uptake of the cells that is necessary to fold EGFP. Within the cultivation a rotation speed of $200 \mathrm{rpm}$ was necessary to produce an acceptable amount of EGFP. Nevertheless the optical density of the suspensions has to be adjusted very carefully to pull down the amount of required oxygen. Better results relating to the oxygen supplying of the cells where acquired by using the microfluidic system for this assay. By permanently circulating the medium within the microfuidic cell a high oxygen transfer could be realized.

\subsubsection{EGFP-reporter gen assay using $\boldsymbol{P}$. pastoris}

The reporter gen assay with genetically modified $P$. pastoris cells was performed in the microtiter plate and in the microfluidic device. The results for the microtiter plate are shown in Image 5. In case of small amounts of methanol up to $0.01 \%$ the fluorescence signals increased up to $48 \mathrm{mV}$ what is twice the signal of the reference. For higher doses of methanol the fluorescence signals increased up to $0.35 \mathrm{~V}$ at a concentration of $0.5 \%$. 


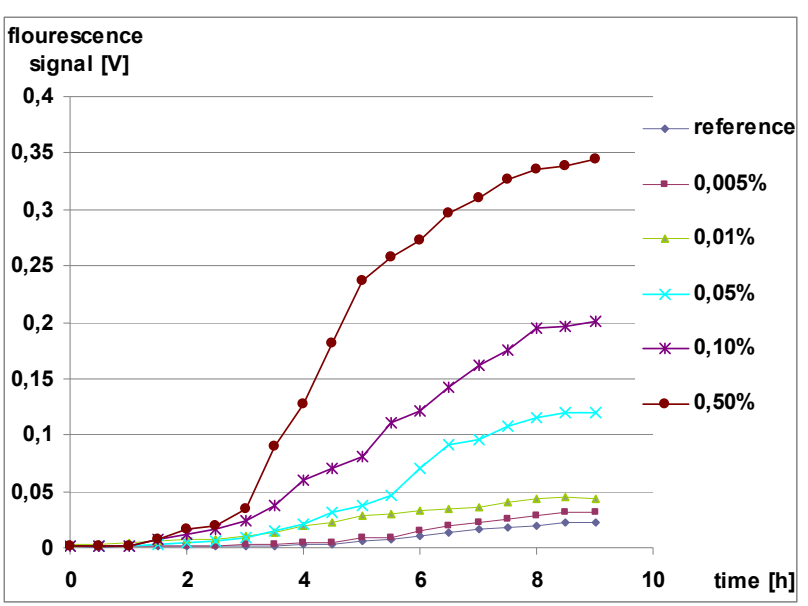

Image 5 Cultivation of $P$. pastoris with different amounts of methanol.

Referring to the growth conditions of Pichia pastoris the short lag-phase at the beginning of the cultivation is a consequence of harvesting the inoculum cultivation during exponential growth phase. For other cultivations with cells harvested during stationary phase the lag phase extended up to $7 \mathrm{~h}$. Like in the cultivation of bacterial cells oxygen uptake was a main problem referring to fluorescence of EGFP. Thus low cell concentrations like OD600 of 0.05 where used to solve that problem. When the cultivation took place in the micro fluidic device cells where grown in higher concentrations because the oxygen transfer through the membrane pump was much higher than in the microtiter plate.

\subsubsection{Human HepG2 cell line stained with Cell Tracker Red and Green}

The fixated HepG2 cells showed fluorescence signals between $0.24 \mathrm{~V}$ with Celltracker Green CMFDA and $0.15 \mathrm{~V}$ with Celltracker Red CMTPX. For the detection of the thickness of the cell layer the fluorescence signal varied between $0.24 \mathrm{~V}$ for a layer consisting out of 3 to 4 cells on top of each other and $0.13 \mathrm{~V}$ for a monolayer of cells using Celltracker Green CMFDA.

\section{Conclusion}

For the fluorescence based online monitoring a robotic guided fluorescence measurement module was developed, which allows the detection of fluorescence signals in microtiter plates and microfluidic systems. This measurement module allows one to work at two different wavelengths (480 / $530 \mathrm{~nm}$ and $570 / 620 \mathrm{~nm})$ and was successfully characterised using EGFP and Rhodamine 6G. Additionaly three cell based assays with bacterial, yeast and human cells were characterized. Therefore cells where grown in microtiter plates or in a microfluidic platform. In most cases the microfluidic system proofed to be the better alternative for the cultivation of the cells compared to microtiter plates. However, the ability to perform many parallel assays in a microtiter plate suggests using these for high throughput assays. Due to the high sensitivity of the fluorescence module further applications could use luciferase based reporter gen assays as well.

\section{Acknowledgements}

We thank the GeSiM mbH for the assistance in spotting technology. This work was financially supported by the European Union and the Free State of Saxony (SAB project UNILOC) and the BMWI (ZIM project RoVitaS).

\section{$5 \quad$ References}

[1] Michael Fritzsche et al., A Cell-based Sensor System for Toxicity Testing Using Multiwavelength Fluorescence Spectroscopy, Analytical Biochemistry 387, no. 2 (April 15, 2009): 271-275.

[2] F. Sommerhage et al., Extracellular Recording of Glycine Receptor Chloride Channel Activity as a Prototype for Biohybrid Sensors, Biosensors and Bioelectronics 26, no. 1 (September 15, 2010): 155-161.

[3] Shimshon Belkin, Microbial Whole-cell Sensing Systems of Environmental Pollutants, Current Opinion in Microbiology 6, no. 3 (June 2003): 206-212.

[4] Sonntag, F.; Gruchow, M.; Wagner, I.; Lindner, G.; Marx, U.: Miniaturisierte humane organtypische Zellund Gewebekulturen. In: BIOspektrum 04.11, 17. Jahrgang, 2011.

[5] Ronald M. Atlas, Handbook of Microbiological Media (Boca Raton, Fla. [u.a.]: CRC Pr., 1993): 741. 